\title{
The progress of High-Energy Physics in Atmosphere (HEPA) achieved with particle physics and nuclear spectroscopy methods
}

\section{A. Chilingaryan}

A.I.Alikhanyan National Lab (Yerevan Physics Institute), Alikhanyan Brothers 2, Yerevan, Armenia

E-mail: chiliearagats.am

\begin{abstract}
At Aragats research station we are consistently applying methods of particle physics and nuclear spectroscopy for revealing details of the operation of electron accelerators evolving just above our heads in the thunderclouds. The newly emerging field of high-energy physics in the atmosphere (HEPA) needs successive application of experimentation paired with the simulation of physical processes with CORSIKA and GEANT4 packages well established in the high-energy physics community. In addition to the minute-long fluxes of high-energy electrons and gamma rays from relativistic runaway electron avalanches (RREA), we discover also the origin of hour-long isotropic fluxes of low-energy gamma rays from the ${ }^{222} \mathrm{Rn}$ progeny gamma radiation. During 12 years of $24 / 7$ monitoring of cosmic ray fluxes, we observed more than half-of-thousand thunderstorm ground enhancements (TGEs). Each cosmic-ray species brings its own special evidence on the structure and strength of the atmospheric electric field. The depletion of muon flux (muon stopping effect) observed simultaneously with the world's largest-ever enhancement of electron and gamma rays' flux at Mt. Lomnicky Stit allows us to estimate the maximum value of the atmospheric electric field. Using a high-precision spectrometer for the measurement of the energy spectra of the natural gamma radiation we discovered a new effect of circulation of radon progeny during thunderstorms. The comparison of electron and gamma ray energy spectra allows us to identify the emerging electrical structures in the atmosphere which makes it possible to accelerate seed electrons from the ambient population of cosmic rays up to $\approx 100 \mathrm{MeV}$. Measuring simultaneously neutron and gamma ray fluxes by a neutron monitor and SEVAN hybrid particle detector we prove the photonuclear origin of the atmospheric neutrons.
\end{abstract}




\section{Introduction}

One of the first particle physicists and researchers of the atmospheric electricity Nobel award winner sir C.T.R. Wilson at the beginning of last century recognized that "a particle may acquire energy corresponding to the greater part of the whole potential difference between the poles of the thundercloud, which may be of the order of $10^{9} \mathrm{~V}$ " (Wilson, 1925).

On August 5, 1963, after more than eight years of difficult negotiations, the United States, the United Kingdom, and the Soviet Union signed the Limited Nuclear Test Ban Treaty that prohibits nuclear weapons tests or other nuclear explosions underwater, in the atmosphere, or in outer space. What is common in these two so different events? The first was a breakthrough in atmospheric physics, the second in keeping the earth's environments clear and both highly impact the discovery of the most energetic natural electron accelerator. The U.S. Air Force launched a series of VEGA satellites designed to verify the conditions of the Treaty; the gamma ray detectors onboard the satellites should monitor nuclear bomb tests by registering intense gamma ray fluxes. However, the Vela gamma-ray detectors picked up high-energy gamma-ray flashes - but they originated not from Earth but from deep space. It was the beginning of an exciting scientific endeavor to find the origin of most violent explosions in the Universe - the gamma ray bursts! These bursts originated in the farthest reaches of the Universe, were later related to the super-luminous supernovae star implosions [1]. The Burst and Transient Source Experiment (BATSE), aboard the Compton Gamma Ray Observatory (CGRO), detects numerous afterglows (lower energy emission accompanied GRBs) and occasionally discovered gamma rays coming from the direction of the earth. Sure, it was not the alerts from A-bomb testing, but the "afterglows" of a much less powerful process - operation of the electron accelerator in the terrestrial atmosphere [2]. The electron accelerator in the upper atmosphere was related to strong thunderstorms in the earth's equatorial regions and was located above 10 $\mathrm{km}$ over the earth's surface. Observing the enhanced fluxes of electrons and gamma rays on the earth's surface turned out to be a rather difficult task. The reviewer of the field [3] noted: "Summing up, after 70 years of numerous theoretical and experimental studies, it is still not clear whether the mechanisms of acceleration of runaway electrons during a thunderstorm or lightning work. In the experiments, usually, only one of the secondary types of cosmic rays was measured, and the number of detected "events with thunderstorm particles" remained very modest (see [4] and references therein).

The situation changed when one of the largest cosmic ray research centers at Mt. Aragats started a research campaign for monitoring fluxes of low-energy cosmic rays. In Fig. 1 we show the Aragats research station of the Yerevan physics institute where during the last decade intensive experimental program was performed for understanding high-energy phenomena in the atmosphere. Since 1943, cosmic ray research on Aragats didn't stop ever during the most difficult years in the history of modern Armenia. At the end of the last century, we finish experiments with large surface arrays registering extensive air showers (EASs), measuring the energy spectra of light and heavy primary nuclei separately [5]. In the old days, the physics of the atmosphere did not seem attractive to us, however, starting to study atmospheric electricity and its modulation effects, we realized that using already existing networks of particle detectors, along with electric field sensors, lightning locators, automatic weather stations and panoramic cameras, we can reach new quality research and investigate thunderclouds with a new type of messengers - cosmic rays. 


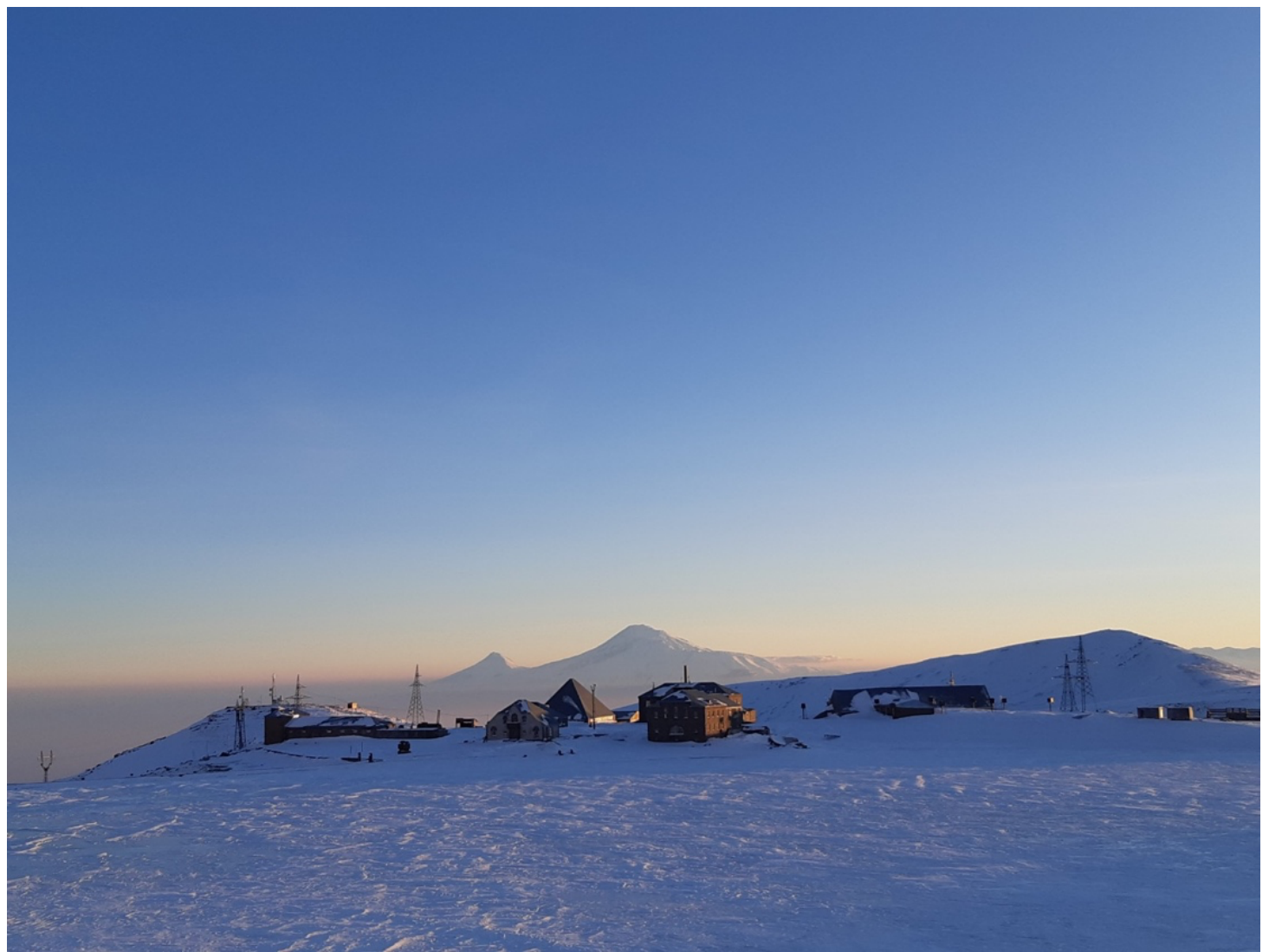

Figure 1. Aragats high-altitude station against the background of the biblical Mount Ararat, to which less than $100 \mathrm{~km}$ in a straight line

The location of our station on the plateau under the southern summit of Mount Aragats near the large mountain lake Kare-lich facilitated the observation of numerous events of thunderstorm ground enhancements (TGEs, [6-8]) especially in the spring, when thunderstorm clouds descend directly to the station. The databases of the CRD are in open access and contain multivariate measurements of hundreds of TGEs, including the time series of count rates of almost all species of secondary cosmic rays, electric and geomagnetic fields strengths, meteorological data, and records of wideband radio emission and interferometer antenna signals. The situation changes when one of the largest cosmic ray research centers at Mt. Aragats starts research campaign for monitoring fluxes of low-energy cosmic rays. In Fig. 1 we show the Aragats research station of the Yerevan physics institute where during the last decade intensive experimental program was performed for understanding high-energy phenomena in the atmosphere. Since 1943, cosmic ray research on Aragats didn't stop ever during the most difficult years in the history of modern Armenia. At the end of the last century, we finish experiments with large surface arrays registering extensive air showers (EASs), measuring the energy spectra of light and heavy primary nuclei separately [5]. In the old days, the physics of the atmosphere did not seem attractive to us, however, starting to study atmospheric electricity and its modulation effects, we realized that using already existing networks of particle detectors, along with electric field sensors, lightning locators, automatic weather stations and panoramic cameras, we can reach a new quality research and investigate thunderclouds with a new type of messengers - cosmic rays. 


\section{Charge structure of the thundercloud and acceleration of electrons}

In Fig. 2 we show the charge structure of the thundercloud, that emerges due to rather complicated processes connected with the friction of different small particles (hydrometeors); as we see in the picture, during the updraft of the warm air and downdraft of the cold air several oppositive charged region emerged. The upper dipole between main negative (MN) and main positive charge regions accelerates seed electrons from an ambient population of secondary electrons created by EASs in the direction to the open space. The lower dipoles between the same main negatively charged region and its mirror on the earth's surface and between the MN and lower positively charged region (LPCR) accelerates electrons in the direction to the earth's surface. Thus, the intracloud electric field modulates the energy spectra of the secondary electrons (the MOS process [9]. However, the MOS process leads to a minor enhancement of the bremsstrahlung gamma rays only, much more intense effect is the relativistic runaway electron avalanches (RREA, [10-12]) appeared in the thunderous atmospheres when electric field surpass a threshold (critical) strength and electrons gain more energy from the electric field than the lost to ionization. The energy of electrons and gamma rays in the avalanche can reach $60-70 \mathrm{MeV}$ and, if the thundercloud is not very high above the earth's surface, the avalanche will be registered by the particle detectors as TGE: abundant fluxes of electrons, gamma rays, and neutrons, exceeding the background flux sometimes more than hundred times. The duration of TGF doesn't exceed milliseconds, only very few gamma rays can reach fast-moving orbiting gamma ray observatories at altitudes $\approx 500 \mathrm{~km}$. The RREA developing in the lower dipole is similar to man-made accelerator experiment; however, the accelerator "beam" has the size of a $\mathrm{km}^{2}$ and its energy is changing each second. Nonetheless, millions and millions of the RREA particles allow to measure energy spectra of TGE electrons and gamma rays, and then recover the high-energy processes in the cloud and discloses charge structures, which supports the origination of the RREA. Thus, the TGE origination can be described as follows:

Cosmic ray electron entering the strong electric field in the cloud, runaway and produce more electrons by the knock-on and other processes; electrons produce gamma rays via bremsstrahlung, gamma rays produce neutrons via photonuclear reactions. 


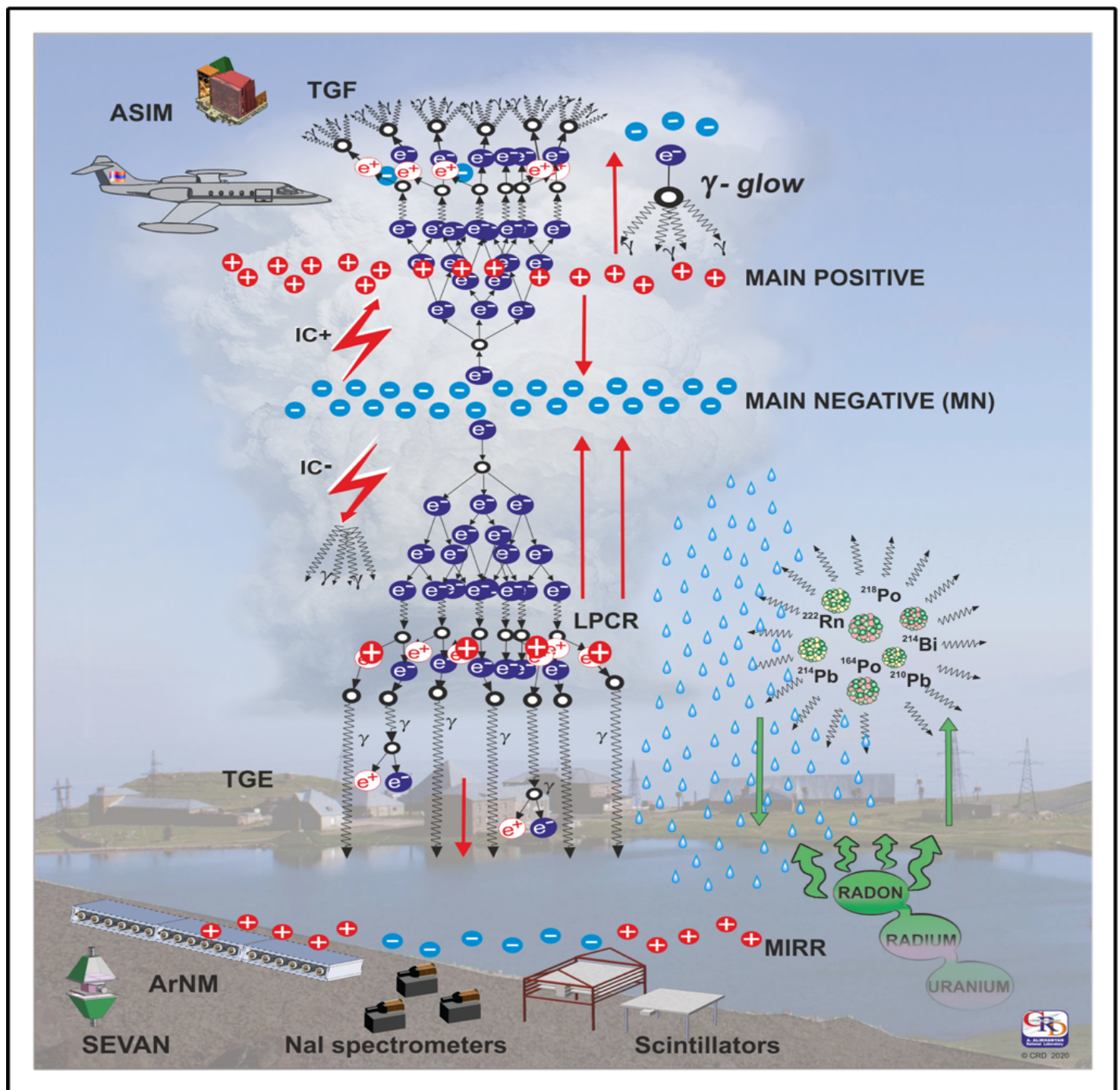

Figure 2. Charge regions of the thundercloud originated TGEs, TGFs, and gamma glows on the earth's surface, in space, and in the troposphere due to RREA development and modification of the electron energy spectrum. Emerging electric fields are shown by red arrows; ${ }^{222} \mathrm{Rn}$ progeny gamma radiation (below $3 \mathrm{MeV}$ ), is shown in green color; also, are shown space-born and surface particle detectors.

All these particles are moving to the earth's surface and produce TGE, seen as large peaks in the time series of count rates of different secondary cosmic ray species above a rather stable background induced by the EASs.

If the electric field is accelerating electrons upward (by the upper dipole) they occasionally reach orbiting gamma-ray observatories and registered by facilities looking to the open space to register gamma bursts coming from very distant regions of the Universe.

Sometimes, balloon and aircraft facilities register gamma rays high in the atmosphere; they called gamma glows because only gamma rays are registered. 


\section{Relativistic Runaway Electron Avalanches and Thunderstorm Ground Enhancements}

The ${ }^{222} \mathrm{Rn}$ progeny gamma radiation in the low energy domain $(<3 \mathrm{MeV})$ significantly extends the time of TGE due to the Radon circulation effect [13]. The near-surface electric field lifted aerosols with attached ${ }^{222} \mathrm{Rn}$ progeny to the atmosphere and precipitation returns it to the earth's surface. In Fig 3 we demonstrate the "long-lasting" TGE: the particle detector with a low energy threshold $(0.8 \mathrm{MeV}$, outdoor $1-\mathrm{cm}$ thick plastic scintillator) registers 4-hour long enhancement; after declining of the storm the low-energy particle enhancement continues hours due to decay of ${ }^{214} \mathrm{~Pb}$ and ${ }^{214} \mathrm{Bi}$ isotopes, see the spectrogram in the right inset to Fig. 3a; the detectors with high energy threshold ( $>3 \mathrm{MeV}, 3$ and $5 \mathrm{~cm}$ thick scintillators) register only high-energy electrons and gamma rays from the RREA process in the atmosphere (extended only a few minutes), see the energy spectra of the electron and gamma ray particles in the left inset to Fig. 3a.

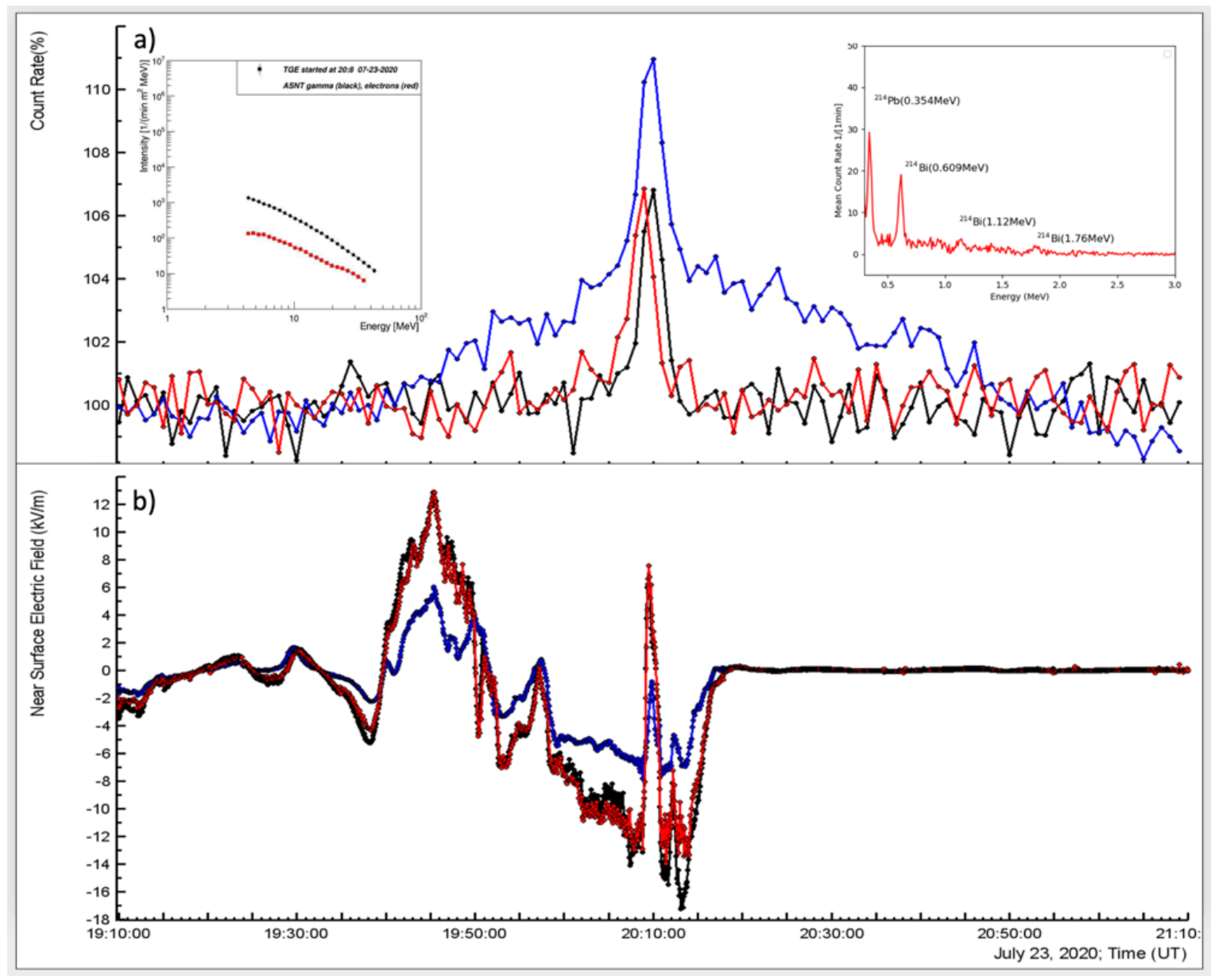

Figure 3. a) One-minute time series of particle detectors count rates: 1- $\mathrm{cm}$ thick, $1 \mathrm{~m}^{2}$ area outdoor plastic scintillator (energy threshold $0.8 \mathrm{MeV}$, blue), the same area 3 and $5 \mathrm{~cm}$ thick plastic scintillators (energy threshold $>3 \mathrm{MeV}$, red and black). In the left inset - the energy spectra of gamma rays (black) and electrons (red) measured at the flux maximum; in the right inset, the spectrogram of low energy flux, the gamma radiation of the ${ }^{222} \mathrm{Rn}$ chain isotopes measured after the storm declined. b) 1-second time series of the nearsurface electric field measured by EFM-100 electric mills.

In Fig. $3 b$ we show the disturbances of the near-surface electric field corresponding to particle 
fluxes shown in Fig. 3a. The low-energy Radon progeny radiation started much earlier and finished much later than a short burst of the high-energy particle flux (coincided with moving of the near-surface electric field to the positive domain at 20:09). This excurses to positive values of the near-surface electric field indicates the emergence of the lower positively charged region at the bottom of the cloud (LPCR) and the strengthening of the electric field in the cloud. The RREA is a threshold process and when the electric field in the cloud surpasses the critical value the avalanche development started. To get insight into cloud charged structure we model particle passage through the electric field with CORSIKA code. We estimate the number of electrons and gamma rays and their maximal energies during traversing the electric field of different strengths and extensions, see Fig. 4. The RREA started high in the atmosphere, where the electric field strength exceeds the critical value by a few tens of percent; with the growing depth in the atmosphere, the surplus to critical energy decreases down until the RREA stops (the critical field strength is proportional to air density).
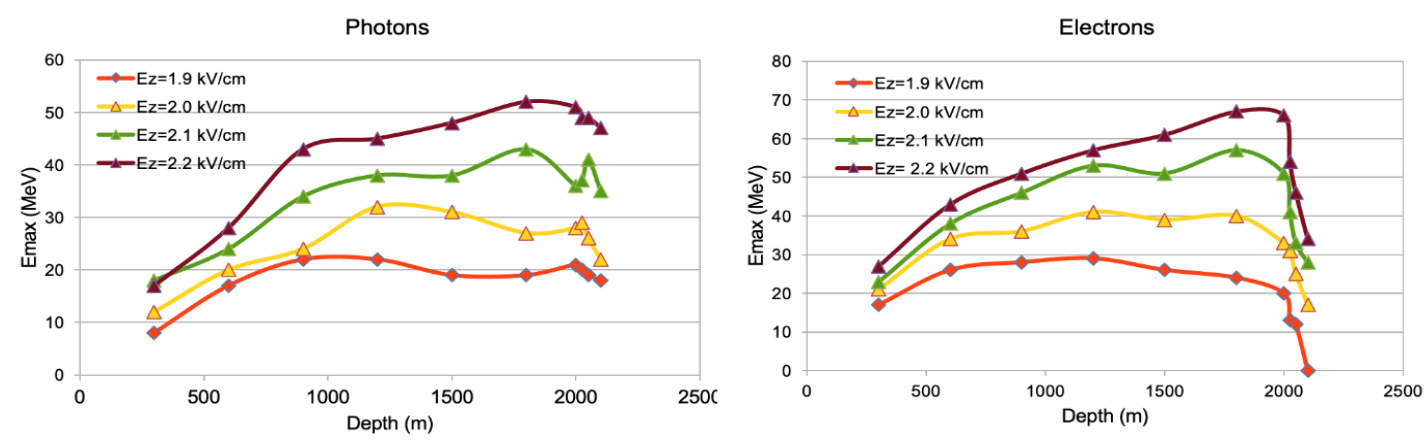

Figure 4. Development of the electromagnetic avalanche in the atmosphere. Avalanche started at $5400 \mathrm{~m}, 2200 \mathrm{~m}$ above the Aragats station (on $5400 \mathrm{~m}$ depth equals 0). The maximum energy of avalanche particles is shown each $300 \mathrm{~m}$. After exiting from the electric field propagation of avalanche particles are followed additionally $200 \mathrm{~m}$ before reaching the station (depth $2200 \mathrm{~m}$ ).

In Fig.4 we show how the maximum energies of the RREA electrons and gamma rays are changing depending on the depth in the atmosphere. If the electric field is high enough (2.2 $\mathrm{kV} / \mathrm{cm}$ ), upper brown curve) the acceleration continues the whole $2 \mathrm{~km}$ propagation and the maximum energy of electrons reaches $70 \mathrm{MeV}$, if the strength of the electric field is lower (1.9 $\mathrm{kV} / \mathrm{cm}$, lower red curve) the RREA is stopping much earlier and maximal electron energy reach only $30 \mathrm{MeV}$. At the exit from the electric field, the electron flux declines very fast due to ionization losses, and by the difference of electron and gamma ray maximum energies, we can estimate the height of the termination of the strong electric field in the cloud [14,15].

\section{Conclusion}

Enhanced particle fluxes registered in space and on the earth's, surface arise in the atmospheric electric fields by the runaway process applied to free electrons from the ambient population of the secondary cosmic rays (EASs). The cosmic ray flux in the thundercloud reveals the charge structure of the cloud that supports RREA and consequent TGE. Large particle detectors and spectrometers operated at Aragats observed 536 TGE events last 12 years, comprising abundant fluxes of electrons, gamma rays, and neutrons. Muons do not multiply in the electric field as electrons; however, electric fields lead to the modernization of their energy spectra and induce a very interesting muon stopping effect, used for the estimation of the maximum energy of the atmospheric electric field [16]. For the estimation of the maximum field, we use the observation of the ever-largest TGE measured at Lomnicky Stit in Slovakia ([17] by SEVAN European particle detector network [18]). 


\section{References}

[1] MacFadyen "Supernovae, Jets, and Collapsars", The Astrophysical Journal (2001) 550, 410.

[2] Fishman, G.J., Bhat, P.N., Mallozzi, et al., Discovery of intense gamma ray flashes of atmospheric origin. Science (1994) 264 (5163), 1313-1316.

[3] Suszcynsky, D.M., Roussel-Dupre, R., Shaw, G., Ground-based search for X-rays generated by thunderstorms and lightning. J. Geophys. Res. (1996) 101, 23,505-23,516.

[4] Chilingarian A., Thunderstorm Ground Enhancements - model and relation to lightning flashes, Journal of Atmospheric and Solar-Terrestrial Physics (2015) 107, 68-76, 2014.

[5] A. Chilingarian, G. Gharagyozyan, G. Hovsepyan, et al., Light and Heavy Cosmic-Ray Energy Spectra as Measured by the MAKET-ANI Detector, Astrophysical Journal (2004) 603, (L29-L32).

[6] Chilingarian A., A. Daryan, K. Arakelyan, et al., Ground-based observations of thunderstormcorrelated fluxes of electrons, gamma rays, and neutrons, Phys Rev D. (2010) 82, 043009.

[7] Chilingarian A., Hovsepyan G., and Hovhannisyan A., Particle bursts from thunderclouds: Natural particle accelerators above our heads, Physical review (2011) D 83, 062001.

[8] A.Chilingarian, G. Hovsepyan, T. Karapetyan, et al., Structure of thunderstorm ground enhancements, PRD (2020) 101, 122004.

[9] Chilingarian A., Mailyan B. and Vanyan L., Recovering of the energy spectra of electrons and gamma rays coming from the thunderclouds,Atmospheric Research (2012) 114-115, 1-16.

[10] Gurevich, A.V., Milikh, G.M., Roussel-Dupre, R., Runaway electron mechanism of air breakdown and preconditioning during a thunder- storm. Phys. Lett. A 1(992) 165, 463-468.

[11] Babich, L.P., et al., Comparison of relativistic runaway electron avalanche rates obtained from Monte Carlo simulations and kinetic equation solution. IEEE Trans. Plasma Sci. (2001) 29 (3), 430.

[12] Dwyer, J.R., A fundamental limit on electric fields in air. Geophys. Res. Lett. (2003) 30 (20), 2055.

[13] A.Chilingarian, G. Hovsepyan, B.Sargsyan, Circulation of Radon progeny in the terrestrial atmosphere during thunderstorms, Geophys. Res. Lett. (2019) 47, e2020GL091155.

[14] A.Chilingarian, G. Hovsepyan, E. Svechnikova, and M. Zazyan, Electrical structure of the thundercloud and operation of the electron accelerator inside it, Astroparticle Physics (2021) 132 102615.

[15] A.Chilingarian, G. Hovsepyan, and M. Zazyan, Measurement of TGE particle energy spectra: An insight in the cloud charge structure, Europhysics letters (2021) https://doi.org/10.1209/02955075/ac0dfa

[16] A.Chilingarian, G. Hovsepyan, G.Karapetyan, and M.Zazyan, Stopping muon effect and estimation of intracloud electric field, Astroparticle Physics (2021) 124, 102505.

[17] A.Chilingarian, T.Karapetyan, H.Hovsepyan, et. al., Maximum strength of the atmospheric electric field, PRD, 2021, PRD (2021) 103, 043021

[18] A.Chilingarian, V. Babayan, T. Karapetyan, et al., The SEVAN Worldwide network of particle detectors: 10 years of operation, Advances in Space Research (2018) 61, 2680-269. 
